\title{
Airy Asymptotics: The logarithmic derivative and its reciprocal
}

\author{
Michael J Kearney ${ }^{1}$ and Richard J Martin ${ }^{2}$ \\ ${ }^{1}$ Faculty of Engineering and Physical Sciences, University of Surrey, \\ Guildford, Surrey, GU2 7XH, United Kingdom \\ ${ }^{2}$ AHL, Man Investments Limited, Sugar Quay, Lower Thames Street, \\ London, EC3R 6DU, United Kingdom
}

July 2009

\begin{abstract}
We consider the asymptotic expansion of the logarithmic derivative of the Airy function $\operatorname{Ai}^{\prime}(z) / \operatorname{Ai}(z)$, and also its reciprocal $\operatorname{Ai}(z) / \operatorname{Ai}^{\prime}(z)$, as $|z| \rightarrow \infty$. In particular, we derive simple, closed-form solutions for the coefficients which appear in these expansions, of interest since they are encountered in a wide variety of problems. The solutions are presented as Mellin transforms of given functions; this fact, together with the methods employed, suggests further avenues for research.
\end{abstract}

PACS: 02.10.Ox; 02.30.Gp; 05.40.Jc

Email:m.j.kearney@surrey.ac.uk 


\section{Introduction}

The Airy function, $\operatorname{Ai}(z)$, which satisfies the second-order, linear differential equation $\operatorname{Ai}^{\prime \prime}(z)-z \operatorname{Ai}(z)=0$, is a well-known and important 'special' function in mathematics and physics. From it one can define two closely related functions, the logarithmic derivative $f(z) \equiv \operatorname{Ai}^{\prime}(z) / \operatorname{Ai}(z)$ and its reciprocal $g(z) \equiv \operatorname{Ai}(z) / \operatorname{Ai}^{\prime}(z)$. These functions, significant in their own right, satisfy the first-order, non-linear Riccati equations $f^{\prime}(z)+f(z)^{2}-z=0$ and $g^{\prime}(z)+z g(z)^{2}-1=0$.

In this paper we are interested in the asymptotic expansion of $f(z)$ and $g(z)$ on the cut-plane $C \backslash(-\infty, 0]$ as $|z| \rightarrow \infty$. It is a relatively simple matter to show that these expansions take the form, ${ }^{1}$

$$
\begin{array}{ll}
f(z) \equiv \frac{\operatorname{Ai}^{\prime}(z)}{\operatorname{Ai}(z)} \sim-z^{1 / 2}\left[1-\sum_{n=1}^{\infty}(-1)^{n} F_{n} z^{-3 n / 2}\right] ; & |\arg (z)|<\pi \\
g(z) \equiv \frac{\operatorname{Ai}(z)}{\operatorname{Ai}^{\prime}(z)} \sim-z^{-1 / 2}\left[1+\sum_{n=1}^{\infty}(-1)^{n} G_{n} z^{-3 n / 2}\right] ; & |\arg (z)|<\pi
\end{array}
$$

but it has been an enduring task to find simple, closed-form (as opposed to recursive) solutions for the coefficients $F_{n}$ and $G_{n}$. Recently, such a solution was found for $F_{n}$; however, the derivation was indirect and consequently somewhat obscure [1]. Here we provide details of a much more direct derivation (which was only sketched in [1] $a$ posteriori), together with the corresponding solution for $G_{n}$, which is rendered accessible in the process.

There are two reasons why the results are of interest. First, these coefficients appear (albeit often in disguised form) in a wide variety of problems (see, e.g., [2,3] for an overview). Specific examples include Brownian motion $[4,5,6,7,8]$, graph theory and combinatorics $[9,10,11,12]$, queueing theory $[13,14]$, algorithm complexity

\footnotetext{
${ }^{1}$ It should be noted that differing notations are used throughout the literature.
} 
theory [15], fluid flow [16], and the enumeration of pointed triangular maps [17]. There is also a subtle link to the rational solutions of the Painlevé II equation [18]. To have an explicit solution in the context of such problems is clearly desirable, e.g., to

study how $F_{n}$ and $G_{n}$ grow with increasing $n$. Second, the solutions obtained have a precise mathematical form (a Mellin transform) which permits an extension of the arithmetic functions $F_{n} \equiv F(z=n)$ and $G_{n} \equiv G(z=n)$ not just to the real line, but to a semi-infinite strip in the complex domain. Such ideas have proven fruitful in the past (see, e.g., [2]) and may offer a fresh perspective on the Airy-Riccati equations, although we do not pursue that line of enquiry in any particular depth here.

\section{Recursion relations and related}

We begin by briefly discussing what is known or easily demonstrated:

(i) Based on the Riccati equations for $f(z)$ and $g(z)$ it follows that $F_{n}$ and $G_{n}$ satisfy quadratic recursion relations of the form [3];

$$
\begin{aligned}
& F_{n}=\left(\frac{3 n-4}{4}\right) F_{n-1}+\frac{1}{2} \sum_{k=1}^{n-1} F_{k} F_{n-k} \\
& G_{n}=\left(\frac{3 n-2}{4}\right) G_{n-1}-\frac{1}{2} \sum_{k=1}^{n-1} G_{k} G_{n-k}
\end{aligned}
$$

where for notational convenience we define $F_{0} \equiv-1$ and $G_{0} \equiv 1$. Iteration of (3) and (4) gives $F_{1}=1 / 4, F_{2}=5 / 32, F_{3}=15 / 64, F_{4}=1105 / 2048, F_{5}=1695 / 1024$ etc and $G_{1}=1 / 4, G_{2}=7 / 32, G_{3}=21 / 64, G_{4}=1463 / 2048, G_{5}=2121 / 1024$ etc. Since $f(z) g(z) \equiv 1$, we also have that $G_{n}=F_{n}+\sum_{k=1}^{k=n-1} F_{k} G_{n-k}$. These recursions provide a computationally efficient way of calculating $F_{n}$ and $G_{n}$ for small values of $n$. They do not, however, provide an explicit, closed-form solution. 
(ii) A different (but equivalent) set of linear recursion relations is obtained by first considering the asymptotic expansions of $\mathrm{Ai}(z)$ and $\operatorname{Ai}^{\prime}(z)$ as $|z| \rightarrow \infty$ [19];

$$
\begin{array}{ll}
\operatorname{Ai}(z) \sim \frac{1}{2 \sqrt{\pi}} z^{-1 / 4} e^{-\frac{2}{3} z^{3 / 2}}\left[1+\sum_{n=1}^{\infty}(-1)^{n} c_{n} z^{-3 n / 2}\right] ; & |\arg (z)|<\pi \\
\operatorname{Ai}^{\prime}(z) \sim-\frac{1}{2 \sqrt{\pi}} z^{1 / 4} e^{-\frac{2}{3} z^{3 / 2}}\left[1-\sum_{n=1}^{\infty}(-1)^{n}\left(\frac{6 n+1}{6 n-1}\right) c_{n} z^{-3 n / 2}\right] ; & |\arg (z)|<\pi
\end{array}
$$

where the coefficient $c_{n}$ is given by,

$$
c_{n}=\frac{\Gamma\left(3 n+\frac{1}{2}\right)}{\Gamma\left(n+\frac{1}{2}\right) 36^{n} n !} .
$$

Through comparison with (1) and (2) these results imply that [3];

$$
\begin{aligned}
& F_{n}=\frac{12 n}{6 n-1} c_{n}-\sum_{k=1}^{n-1} c_{k} F_{n-k} \\
& G_{n}=\frac{12 n}{6 n-1} c_{n}+\sum_{k=1}^{n-1} \frac{6 k+1}{6 k-1} c_{k} G_{n-k} .
\end{aligned}
$$

In a sense these recursions are 'less fundamental' than those derived from the Riccati perspective. Certainly (3) and (4) are encountered more often in practice.

(iii) Closed-form solutions for $F_{n}$ and $G_{n}$ can be obtained using (5) and (6), but they are cumbersome and certainly not deserving of the adjective simple. Thus, by writing $f(z)=(d / d z) \ln \operatorname{Ai}(z)$ and using (5) in a formal sense to expand the logarithm, it follows that, 


$$
F_{n+1}=\left(\frac{3 n}{2}\right)\left[c_{n}-\frac{1}{2} \sum_{\substack{k, l \geq 1 \\ k+l=n}} c_{k} c_{l}+\frac{1}{3} \sum_{\substack{k, l, m \geq 1 \\ k+l+m=n}} c_{k} c_{l} c_{m}-\ldots+\frac{(-1)^{n+1}}{n} c_{1}^{n}\right]
$$

In the same vein, recognising that $g(z)=z^{-1}(d / d z) \ln \left[-\mathrm{Ai}^{\prime}(z)\right]$ and using (6), one can show by expanding the logarithm that,

$$
\begin{aligned}
G_{n+1}=\left(\frac{3 n}{2}\right) & {\left[\left(\frac{6 n+1}{6 n-1}\right) c_{n}+\frac{1}{2} \sum_{\substack{k, l \geq 1 \\
k+l=n}}\left(\frac{6 k+1}{6 k-1}\right)\left(\frac{6 l+1}{6 l-1}\right) c_{k} c_{l}\right.} \\
& \left.+\frac{1}{3} \sum_{\substack{k, l, m \geq 1 \\
k+l+m=n}}\left(\frac{6 k+1}{6 k-1}\right)\left(\frac{6 l+1}{6 l-1}\right)\left(\frac{6 m+1}{6 m-1}\right) c_{k} c_{l} c_{m}+\ldots+\frac{1}{n}\left(\frac{7}{5}\right)^{n} c_{1}^{n}\right] .
\end{aligned}
$$

Little attention seems to have been paid to these expressions, probably because they are awkward to evaluate (after combining terms through symmetry, the number of distinct terms is given by the partition function $p(n)$ which increases rapidly with $n$ ). As a result, (10) and (11) are not particularly useful or informative.

\section{Main results and method of derivation}

In what follows we will approach the problem differently, based on the line of reasoning sketched in [1], and further inspired by [2]. This involves focussing on the analytic structure of $f(z)$ and $g(z)$. By doing so we shall prove that for $n \geq 1$,

$$
\begin{aligned}
& F_{n}=\frac{3}{2 \pi^{2}} \int_{0}^{\infty} \frac{x^{3(n-1) / 2}}{\operatorname{Ai}^{2}(x)+\mathrm{Bi}^{2}(x)} d x \\
& G_{n}=\frac{3}{2 \pi^{2}} \int_{0}^{\infty} \frac{x^{(3 n-1) / 2}}{\operatorname{Ai}^{\prime 2}(x)+\mathrm{Bi}^{\prime 2}(x)} d x
\end{aligned}
$$

where $\operatorname{Bi}(z)$ is the second, linearly independent, solution of the Airy equation. These results are concise and powerful, especially when it comes to studying the $n \rightarrow \infty$ 
limit. Moreover, each is expressed (to within a change of variable) as the Mellin transform of a given function. This means that by replacing the integer $n$ with a complex variable $z$ one has a natural extension of the arithmetic functions $F_{n} \equiv F(z=n)$ and $G_{n} \equiv G(z=n)$ to the complex domain, provided that the range of $z$ satisfies $\alpha<\operatorname{Re}(z)<\beta$ so that the integrals converge. In this instance $\alpha=1 / 3$ for $F(z)$ and $\alpha=-1 / 3$ for $G(z)$, whilst $\beta=\infty$ for both.

We consider the derivation of (12) first and note as a starting point that the zeros of the function $\operatorname{Ai}(z)$ in the complex plane are restricted to the negative real axis [19]. Thus the function $z^{-1 / 2} \mathrm{Ai}^{\prime}(z) / \mathrm{Ai}(z)$ is analytic everywhere on the cut-plane $C \backslash(-\infty, 0]$. We may therefore choose to write for some fixed, finite (but otherwise arbitrary) value of $N$,

$$
\frac{1}{z^{1 / 2}} \frac{\operatorname{Ai}^{\prime}(z)}{\operatorname{Ai}(z)}=\sum_{k=0}^{N}(-1)^{k} F_{k} z^{-3 k / 2}+R_{N}^{(f)}(z) ; \quad|\arg (z)|<\pi
$$

where $F_{0} \equiv-1$ and $F_{k}$ for $1 \leq k \leq N$ is chosen from the sequence generated by (3) or (8). Key to the following argument is the fact that, by construction, as $|z| \rightarrow \infty$ the unknown 'remainder' term $R_{N}^{(f)}(z)$ has the property,

$$
R_{N}^{(f)}(z)=O\left(|z|^{-3(N+1) / 2}\right) ; \quad|\arg (z)|<\pi
$$

This is a consequence of (1) being a valid asymptotic expansion everywhere on $C \backslash(-\infty, 0]$, which implies that (15) holds by definition. Next we multiply each term in (14) by $z^{(3 n-2) / 2}$, with $1 \leq n<N$, and then integrate along a circular arc, orientated anti-clockwise, of radius $\rho$, which starts at $\rho e^{-i 2 \pi / 3}$ and ends at $\rho e^{i 2 \pi / 3}$. Thus,

$$
\begin{aligned}
\int_{C} \frac{1}{z^{1 / 2}} \frac{\operatorname{Ai}^{\prime}(z)}{\operatorname{Ai}(z)} z^{3 n / 2} \frac{d z}{z} \\
=\int_{C} \sum_{k=0}^{N}(-1)^{k} F_{k} z^{-3 k / 2} z^{3 n / 2} \frac{d z}{z}+\int_{C} R_{N}^{(f)}(z) z^{3 n / 2} \frac{d z}{z} .
\end{aligned}
$$


The first term(s) on the right hand side of (16) may be integrated directly by making the substitution $z^{3 / 2}=\rho e^{i \theta}$;

$$
\begin{aligned}
\int_{C} \sum_{k=0}^{N}(-1)^{k} F_{k} z^{-3 k / 2} z^{3 n / 2} \frac{d z}{Z} & \\
= & \frac{2 i}{3} \sum_{k=0}^{N}(-1)^{k} F_{k} \rho^{n-k} \int_{-\pi}^{\pi} e^{i(n-k) \theta} d \theta=\frac{4 \pi i}{3}(-1)^{n} F_{n} .
\end{aligned}
$$

For the 'remainder' term on the right hand side of (16) we make the observation, using the property outlined in (15), that in the limit $\rho \rightarrow \infty$,

$$
\left|\int_{C} R_{N}^{(f)}(z) z^{3 n / 2} \frac{d z}{z}\right|<\frac{C_{N}}{\rho^{3(N-n+1) / 2}}
$$

for some $N$-dependent constant $C_{N}$. Clearly for $1 \leq n<N$ this term vanishes as $\rho \rightarrow \infty$, and since we will shortly take the limit $\rho \rightarrow \infty$, we can drop this term hereafter. The last step before letting $\rho \rightarrow \infty$ is to consider the integration contour on the left hand side of (16). Since the integrand is analytic on the cut-plane, it is possible to deform the contour onto one which runs along two rays; the first from $\rho e^{-i 2 \pi / 3}$ to 0 and the second from 0 to $\rho e^{i 2 \pi / 3}$ (by a limiting procedure one can include $z=0$ ). Now letting $\rho \rightarrow \infty$ and rearranging one has that,

$$
F_{n}=(-1)^{n} \frac{3}{4 \pi i} \int \frac{\operatorname{Ai}^{\prime}(z)}{\operatorname{Ai}(z)} z^{3(n-1) / 2} d z
$$

where the wedge contour $>$ runs from $\infty e^{-i 2 \pi / 3}$ to 0 and from 0 to $\infty e^{i 2 \pi / 3}$. Since the choice of $N$ was arbitrary, it follows that (19) is valid for all $n \geq 1$. 
To show that (19) leads to (12) we make two further observations. Taking the parameterisation $z=x e^{-i 2 \pi / 3}$ on the lower ray and $z=x e^{i 2 \pi / 3}$ on the upper ray (note that $x$ is a real variable) gives,

$$
F_{n}=\frac{3}{4 \pi i} \int_{0}^{\infty}\left[e^{-i 2 \pi / 3} \frac{\operatorname{Ai}^{\prime}\left(e^{-i 2 \pi / 3} x\right)}{\operatorname{Ai}\left(e^{-i 2 \pi / 3} x\right)}-e^{i 2 \pi / 3} \frac{\operatorname{Ai}^{\prime}\left(e^{i 2 \pi / 3} x\right)}{\operatorname{Ai}\left(e^{i 2 \pi / 3} x\right)}\right] x^{3(n-1) / 2} d x
$$

Using the important and very useful identity [19],

$$
\operatorname{Ai}\left(z e^{ \pm 2 \pi i / 3}\right)=\frac{1}{2} e^{ \pm \pi i / 3}[\operatorname{Ai}(z) \mp i \operatorname{Bi}(z)]
$$

this can be simplified to give,

$$
\begin{gathered}
F_{n}=\frac{3}{4 \pi i} \int_{0}^{\infty}\left[\frac{\operatorname{Ai}^{\prime}(x)+i \mathrm{Bi}^{\prime}(x)}{\operatorname{Ai}(x)+i \operatorname{Bi}(x)}-\frac{\operatorname{Ai}^{\prime}(x)-i \mathrm{Bi}^{\prime}(x)}{\operatorname{Ai}(x)-i \operatorname{Bi}(x)}\right] x^{3(n-1) / 2} d x \\
=\frac{3}{2 \pi} \int_{0}^{\infty}\left[\frac{\operatorname{Ai}(x) \operatorname{Bi}^{\prime}(x)-\operatorname{Ai}^{\prime}(x) \operatorname{Bi}(x)}{\operatorname{Ai}^{2}(x)+\operatorname{Bi}^{2}(x)}\right] x^{3(n-1) / 2} d x .
\end{gathered}
$$

Finally, noting the form of the Wronskian [19],

$$
W\{\operatorname{Ai}(z), \operatorname{Bi}(z)\} \equiv \operatorname{Ai}(z) \operatorname{Bi}^{\prime}(z)-\operatorname{Ai}^{\prime}(z) \operatorname{Bi}(z)=\frac{1}{\pi}
$$

this reduces to the desired result (12). The reason for the original choice of contour is now clear; it enables the identity (21) to be exploited in the manner indicated.

Having derived (12), the derivation of (13) is carried out in much the same manner. Noting that the zeros of the function $\operatorname{Ai}^{\prime}(z)$ are also restricted to the negative real axis [19], this time we consider the function $z^{1 / 2} \mathrm{Ai}(z) / \mathrm{Ai}^{\prime}(z)$ on the cut-plane $C \backslash(-\infty, 0]$ and write, 


$$
z^{1 / 2} \frac{\operatorname{Ai}(z)}{\operatorname{Ai}^{\prime}(z)}=\sum_{k=0}^{N}(-1)^{k+1} G_{k} z^{-3 k / 2}+R_{N}^{(g)}(z) ; \quad|\arg (z)|<\pi
$$

where $G_{0} \equiv 1$ and $G_{k}$ for $1 \leq k \leq N$ is chosen from the sequence generated by (4) or (9). The calculation proceeds exactly as before using the same logic to the point where one has established that for $n \geq 1$,

$$
G_{n}=(-1)^{n+1} \frac{3}{4 \pi i} \int \frac{\operatorname{Ai}(z)}{\operatorname{Ai}^{\prime}(z)} z^{(3 n-1) / 2} d z
$$

Again, taking the parameterisation $z=x e^{-i 2 \pi / 3}$ on the lower ray and $z=x e^{i 2 \pi / 3}$ on the upper ray, this gives,

$$
G_{n}=\frac{3}{4 \pi i} \int_{0}^{\infty}\left[e^{i 4 \pi / 3} \frac{\operatorname{Ai}\left(e^{i 2 \pi / 3} x\right)}{\operatorname{Ai}^{\prime}\left(e^{i 2 \pi / 3} x\right)}-e^{-i 4 \pi / 3} \frac{\operatorname{Ai}\left(e^{-i 2 \pi / 3} x\right)}{\operatorname{Ai}^{\prime}\left(e^{-i 2 \pi / 3} x\right)}\right] x^{(3 n-1) / 2} d x
$$

Using (21) this simplifies to,

$$
G_{n}=\frac{3}{2 \pi} \int_{0}^{\infty}\left[\frac{\operatorname{Ai}(x) \operatorname{Bi}^{\prime}(x)-\operatorname{Ai}^{\prime}(x) \operatorname{Bi}(x)}{\operatorname{Ai}^{\prime 2}(x)+\operatorname{Bi}^{\prime 2}(x)}\right] x^{(3 n-1) / 2} d x
$$

and using (23) this reduces to (13). It should be emphasised that (12) and (13) are exact for all $n \geq 1$. To satisfy our curiosity, we have checked them both numerically up to $n=20$.

\section{Comments and further analysis}

The derivations complete, we offer the following observations and analysis:

(i) The symmetry exhibited between $\mathrm{Ai}(z)$ and $\operatorname{Bi}(z)$ in (12) and (13) is revealing. At one level it is an expression of the fact that the functions $\operatorname{Bi}^{\prime}(z) / \operatorname{Bi}(z)$ and $\operatorname{Bi}(z) / \operatorname{Bi}^{\prime}(z)$ obey the same Riccati equations as $\operatorname{Ai}^{\prime}(z) / \operatorname{Ai}(z)$ and $\operatorname{Ai}(z) / \operatorname{Ai}^{\prime}(z)$ 
respectively, albeit with different boundary conditions. The corresponding asymptotic expansions as $|z| \rightarrow \infty$ are given by,

$$
\begin{array}{ll}
\frac{\operatorname{Bi}^{\prime}(z)}{\operatorname{Bi}(z)} \sim z^{1 / 2}\left[1-\sum_{n=1}^{\infty} F_{n} z^{-3 n / 2}\right] ; & |\arg (z)|<\frac{\pi}{3} \\
\frac{\operatorname{Bi}(z)}{\operatorname{Bi}^{\prime}(z)} \sim z^{-1 / 2}\left[1+\sum_{n=1}^{\infty} G_{n} z^{-3 n / 2}\right] ; & |\arg (z)|<\frac{\pi}{3}
\end{array}
$$

and, although these are different to (1) and (2), they involve the same coefficients $F_{n}$ and $G_{n}$. For completeness, the expansions (28) and (29) can also be obtained from the asymptotic expansions of $\operatorname{Bi}(z)$ and $\operatorname{Bi}^{\prime}(z)$ as $|z| \rightarrow \infty[19]$,

$$
\begin{aligned}
& \operatorname{Bi}(z) \sim \frac{1}{\sqrt{\pi}} z^{-1 / 4} e^{\frac{2}{3} z^{3 / 2}}\left[1+\sum_{n=1}^{\infty} c_{n} z^{-3 n / 2}\right] ; \quad|\arg (z)|<\frac{\pi}{3} \\
& \operatorname{Bi}^{\prime}(z) \sim \frac{1}{\sqrt{\pi}} z^{1 / 4} e^{\frac{2}{3^{3 / 2}}}\left[1-\sum_{n=1}^{\infty}\left(\frac{6 n+1}{6 n-1}\right) c_{n} z^{-3 n / 2}\right] ; \quad|\arg (z)|<\frac{\pi}{3}
\end{aligned}
$$

with $c_{n}$ given by (7). At a deeper level, this symmetry suggests that the extended functions $F(z)$ and $G(z)$ (which can be inverted using the inverse Mellin transform) are a fundamental manifestation of the Airy-Riccati equations themselves rather than any particular solution thereof. As such, they are probably deserving of further study. One is struck by analogies with other functions, the simplest being the Gamma function whose integral representation $\Gamma(z)=\int_{0}^{\infty} x^{z-1} e^{-x} d x$ is of Mellin type and for which $\Gamma_{n} \equiv \Gamma(z=n)$ has the elementary recursive form $\Gamma_{n}=(n-1) \Gamma_{n-1}$.

(ii) The solutions for $F_{n}$ and $G_{n}$ provide an efficient way of studying the $n \rightarrow \infty$ limit. The starting observation (easy to prove) is that as $n \rightarrow \infty$ the integrands of both (12) and (13) reach a maximum at $x_{m}=(3 n / 4)^{2 / 3}+O\left(n^{-1 / 3}\right)$. Using elementary 
asymptotic methods an expansion can thus be obtained based on (30) and (31) alone, since $\operatorname{Bi}\left(x_{m}\right)>>\operatorname{Ai}\left(x_{m}\right)$ and $\operatorname{Bi}^{\prime}\left(x_{m}\right)>>\left|\operatorname{Ai}^{\prime}\left(x_{m}\right)\right|$. Retaining the first three terms of (30) and (31) in conjunction with (12) and (13) is sufficient to establish that,

$$
\begin{aligned}
& F_{n}=\frac{1}{\pi}\left(\frac{3}{4}\right)^{n}(n-1) !\left[1-\frac{5}{18 n}-\frac{335}{648 n^{2}}+O\left(\frac{1}{n^{3}}\right)\right] \\
& G_{n}=\frac{1}{\pi}\left(\frac{3}{4}\right)^{n}(n-1) !\left[1+\frac{7}{18 n}+\frac{553}{648 n^{2}}+O\left(\frac{1}{n^{3}}\right)\right] .
\end{aligned}
$$

The leading terms agree with those presented in [3,4,5], whilst the second term in (32) appears in [8]. Those studies were based on a delicate analysis of the recursion relations (3), (4), (8) and (9). Evaluating the higher order terms is much easier using the present approach.

(iii) One can define integer sequences through $f_{n} \equiv 2^{3 n-1} F_{n}$ and $g_{n} \equiv 2^{3 n-1} G_{n}$. Thus $f_{1}=1, f_{2}=5, f_{3}=60, f_{4}=1105, f_{5}=27120$ etc and $g_{1}=1, g_{2}=7$, $g_{3}=84, g_{4}=1463, g_{5}=33936$ etc. With reference to (3) and (4) these sequences are generated recursively;

$$
\begin{array}{ll}
f_{n}=(6 n-8) f_{n-1}+\sum_{k=1}^{n-1} f_{k} f_{n-k} ; & f_{0} \equiv-\frac{1}{2} \\
g_{n}=(6 n-4) g_{n-1}-\sum_{k=1}^{n-1} g_{k} g_{n-k} ; & g_{0} \equiv \frac{1}{2}
\end{array}
$$

The sequence for $f_{n}$ is documented as A062980 in [20] and it appears explicitly in several of the problems discussed earlier $[12,15,16,17]$. As a consequence of (12) and (13) we have the following solutions (after a simple change of variable); 


$$
\begin{aligned}
& f_{n}=\frac{1}{\pi^{2}} \int_{0}^{\infty} \frac{x^{n-4 / 3}}{\operatorname{Ai}^{2}\left(\frac{1}{4} x^{2 / 3}\right)+\operatorname{Bi}^{2}\left(\frac{1}{4} x^{2 / 3}\right)} d x \\
& g_{n}=\frac{1}{4 \pi^{2}} \int_{0}^{\infty} \frac{x^{n-2 / 3}}{\operatorname{Ai}^{\prime 2}\left(\frac{1}{4} x^{2 / 3}\right)+\mathrm{Bi}^{\prime 2}\left(\frac{1}{4} x^{2 / 3}\right)} d x .
\end{aligned}
$$

These results make a striking impression. As an exercise in analytic combinatorics [21] there is also the prospect that solutions to other integer sequences generated by self-convolutive recursions might be found using the same basic technique. This will be pursued elsewhere. The growth of $f_{n}$ and $g_{n}$ as $n \rightarrow \infty$ follows directly from (32) and (33) after scaling by a factor of $2^{3 n-1}$.

\section{Conclusions}

In summary, simple, closed-form solutions (expressed as Mellin transforms of given functions) have been derived for the coefficients entering the asymptotic expansion of the logarithmic derivative of the Airy function $\operatorname{Ai}^{\prime}(z) / \operatorname{Ai}(z)$ and its reciprocal $\operatorname{Ai}(z) / \operatorname{Ai}^{\prime}(z)$ as $|z| \rightarrow \infty$. This is of direct interest in the context of a variety of problems. In addition, several avenues for further research have been suggested, extending both the results and the method used to obtain them. As to why the main results in this paper are not already well-established, one can speculate that a natural reluctance to believe that the calculation would yield a useful answer has prevented researchers from trying. With the benefit of hindsight the analysis is quite straightforward, so extensions in other directions should be perfectly feasible. 


\section{References}

[1] Kearney M J, Majumdar S N and Martin R J 2007 J. Phys. A: Math. Theor. 40 F863

[2] Flajolet P and Louchard G 2001 Algorithmica 31361

[3] Janson S 2007 Probability Surveys 480

[4] Takacs L 1991 Adv. Appl. Probab. 23557

[5] Takacs L 1992 Ann. Appl. Probab. 2435

[6] Perman M and Warner J A 1996 Ann. Appl. Probab. 61091

[7] Majumdar S N and Comtet A 2005 J. Stat. Phys. 119777

[8] Janson S and Louchard G 2007 Elec. J. Probab. 121600

[9] Wright E M 1977 J. Graph Theor. 1317

[10] Wright E M 1980 J. Graph Theor. 4393

[11] Spencer J 1997 Comm. Pure Appl. Math. 50291

[12] Janson S 2003 Random Structures Algorithms 22337

[13] Kearney M J 2004 J. Phys. A: Math. Gen. 378421

[14] Kearney M J 2006 Phys. Rev. E. 74061115

[15] Flajolet P, Poblete P and Viola A 1998 Algorithmica 22490

[16] Wilkinson M, Mehlig B, Ostlund S and Duncan K P 2007 Phys. Fluids 19 113303

[17] Vidal S A 2007; arXiv:0706.0969

[18] Iwasaki K, Kajiwara K and Nakamura T 2002 J. Phys. A: Math. Gen. 35 L207

[19] Abramowitz M and Stegun I A 1973 Handbook of Mathematical Functions (New York: Dover)

[20] Sloane N J A Ed., The On-Line Encyclopedia of Integer Sequences, www.research.att.com/ njas/sequences/A062980

[21] Flajolet P and Sedgewick R 2009 Analytic Combinatorics (New York: Cambridge University Press) 